\title{
Coma mixedematoso como presentación de hipotiroidismo
}

\section{(Myxedema coma as presentation of hypothyroidism)}

\author{
Roberto Gabriel Albín-Cano, Alexis Parada-Valdés, José O. Ramírez-Quintana
}

\section{Resumen}

Se presenta un caso de una paciente de 69 años de edad y sin antecedentes personales o familiares de patología tiroidea alguna, que asiste con un estado convulsivo de aparición súbita, de hemicuerpo derecho y relajación esfinteriana, asociado, además, a trastornos del estado de conciencia, bradicardia, bradipnea y edema de difícil Godet en miembros inferiores. Los exámenes complementarios mostraron acidosis respiratoria, hiposecreción de T4 y elevación de la hormona estimulante de la tiroides por retroalimentación negativa. Estos resultados corroboraron el diagnóstico presuntivo de coma mixedematoso. Se emprendió el tratamiento hormonal correspondiente con levotiroxina, al cual respondió satisfactoriamente y fue egresada a los 24 días de su ingreso, pendiente de valoración por el especialista de endocrinología.

Descriptores: coma mixedematoso, hipotiroidismo, hormonas tiroideas, emergencia endocrina.

\section{Abstract}

We present the case of a 69-year-old female patient with no personal or family history of any thyroid disease, who attends with a convulsive state of sudden onset, right hemibody and sphincter relaxation, also associated with disorders of the state of consciousness, bradycardia, bradypnea and edema of difficult godet in lower limbs. Complementary analyses showed respiratory acidosis, hyposecretion of T4 and elevation of the thyroid stimulating hormone by negative feedback. These results corroborated the presumptive diagnosis of myxedema coma. The

Afiliación de los autores: Departamento de Medicina Interna. Hospital ClínicoQuirúrgico "Freyre de Andrade". Facultad de Ciencias Médicas "Gral. Calixto García Îñiguez". Universidad de Ciencias Médicas de La Habana. La Habana, Cuba.

Conflicto de intereses: no se tiene conflicto de interés por declarar.

凶alexisparada1803@gmail.com

ISSN 0001-6012/2019/61/4/187-189

Acta Médica Costarricense, (ㅇ 2019

Colegio de Médicos y Cirujanos

de Costa Rica corresponding hormonal treatment with levothyroxine was undertaken, to which it responded satisfactorily and was discharged 24 days after admission, pending evaluation by the endocrinology specialist for an outpatient hormone replacement therapy.

Keywords: Myxedema Coma, Hypothyroidism, thyroid hormones, endocrine emergency.

Fecha recibido: 03 de junio 2019 Fecha aprobado: 19 de septiembre 2019

Las hormonas tiroideas son esenciales para el desarrollo y diferenciación adecuada de todas las células del cuerpo humano. Estas hormonas intervienen regulando el metabolismo y el funcionamiento de órganos primordiales, tienen acción calorígena y termorreguladora, aumentan el consumo de oxígeno y la tasa de ventilación, incrementan la frecuencia cardíaca, acrecentando a su vez el gasto cardíaco y participando, además, en los procesos de contracción muscular y motilidad intestinal. ${ }^{1}$ La hiposecreción de estas hormonas conlleva al hipotiroidismo, el cual, sin tratamiento adecuado, se traduciría en un coma mixedematoso, provocando cambios metabólicos como bradipnea, bradicardia, relajación muscular e hipotermia.

El coma mixedematoso puede ser desencadenado por un proceso infeccioso, generalmente respiratorio y urinario. De igual modo, aunque en menor frecuencia, puede desencadenarse por medicamentos que deprimen el sistema nervioso central, entre ellos los betabloqueadores, el litio, que disminuye la liberación de hormonas tiroideas, la amiodarona y el midazolam. Otros factores son la exposición prolongada al frío, así como todas las situaciones que aumentan las necesidades energéticas. ${ }^{2,3}$ Aún con un diagnóstico temprano y tratamiento adecuado, la mortalidad es de un 50-60\%, lo que indica el peligro., Debido al avance de las capacidades diagnósticas se ha logrado reducir esos valores hasta un 20-40 \%. ${ }^{6}$ Se presenta con mayor frecuencia en mujeres que en hombres, con una incidencia de aproximadamente 0,22 por cada 1 millón de personas en el año, y se manifiesta casi de manera exclusiva en personas de 60 años o mayores. ${ }^{7.9}$ Usualmente, el diagnóstico es tardío, debido a que sus síntomas son inespecíficos y pueden confundirse con insuficiencia cardíaca, alteraciones del sistema nervioso central e insuficiencia renal crónica descompensada. La presentación de este caso pretende evidenciar la particularidad especial del coma mixedematoso en ausencia de antecedentes personales de hipotiroidismo, lo que dificulta el diagnóstico de dicha afección y resulta una desventaja para el tratamiento. Presenta 
gran relevancia por ser una complicación poco frecuente y un desafío en el actuar médico.

\section{Presentación del caso}

Paciente femenina de 69 años de edad con antecedentes patológicos personales de hipertensión arterial de 4 años de evolución, tratada con 1 tableta diaria de metildopa, enalapril y amlodipino; enfermedad cerebrovascular hemorrágica de 4 años de evolución, y un tumor abdominal uterino diagnosticado hace 1 año; ella es referida a nuestro centro por presentar un estado convulsivo de aparición súbita en hemicuerpo derecho, acompañado de relajación esfinteriana vesical, pérdida de conciencia y sialorrea. Examen físico: Se evidenció fascies mixedematosa, tejido celular subcutáneo infiltrado en cara y miembros inferiores, consistencia dura, y sin presencia de godet. Frecuencia respiratoria en 12 respiraciones / minuto, frecuencia cardíaca en 52 latidos / minuto, macroglosia marcada. Paciente estuporosa, con débil respuesta a los estímulos verbales y dolorosos, disartria. Presencia de signo de Babinski en miembro inferior derecho, hipotonía e hiporreflexia generalizada. Sin signos meníngeos. Tomografía axial computarizada con atrofia cortical y subcortical.

Los exámenes complementarios reflejaron anemia secundaria a la disminución de los requerimientos de oxígeno y de la eritropoyetina, hipoxemia e hipercapnia, expresando una acidosis respiratoria. Los niveles elevados de creatinina y ácido úrico implican un deterioro nefrítico importante, debido a disminución del flujo plasmático y filtrado glomerular, hipoglicemia, hiposecreción de T4 y elevación de la hormona estimulante de la tiroides por retroalimentación negativa (Cuadro 1).

\begin{tabular}{|lcc|}
\hline $\begin{array}{c}\text { Cuadro 1. Exámenes complementarios realizados a la } \\
\text { paciente al ingreso en el Hospital Clínico-Quirúrgico } \\
\text { "Freyre de Andrade" }\end{array}$ \\
\hline $\begin{array}{l}\text { Exámenes } \\
\text { complementarios }\end{array}$ & Resultados & Rangos normales \\
\hline Hemoglobina & $8,2 \mathrm{mg} / \mathrm{dl}$ & $12-16 \mathrm{mg} / \mathrm{dL}$ \\
$\mathrm{pH}$ arterial & 7,09 & $7,35-7,45$ \\
$\mathrm{pCO}_{2}$ & $71,9 \mathrm{mmHg}$ & $35-45 \mathrm{mmHg}$ \\
$\mathrm{pO}_{2}$ & $43 \mathrm{mmHg}$ & $80-100 \mathrm{mmHg}$ \\
$\mathrm{HCO}_{3}$ & $21,6 \mathrm{mmol} / \mathrm{L}$ & $21-28 \mathrm{mEg} / \mathrm{L}$ \\
$\mathrm{Glicemia}$ & $2,87 \mathrm{mmol} / \mathrm{L}$ & $3,2-6,2 \mathrm{mmol} / \mathrm{L}$ \\
$\mathrm{Creatinina}$ & $255,16 \mathrm{umol} / \mathrm{L}$ & $50-132 \mathrm{umol} / \mathrm{L}$ \\
Ácido úrico & $436,7 \mathrm{umol} / \mathrm{L}$ & $142-339 \mathrm{umol} / \mathrm{L}$ \\
$\mathrm{TGP}$ & $23,9 \mathrm{u} / \mathrm{L}$ & $5-40 \mathrm{U} / \mathrm{L}$ \\
$\mathrm{TGO}$ & $27,3 \mathrm{u} / \mathrm{L}$ & $5-40 \mathrm{U} / \mathrm{L}$ \\
Albúmina & $37,2 \mathrm{~g} / \mathrm{L}$ & $34-47 \mathrm{~g} / \mathrm{L}$ \\
$\mathrm{T}_{4}$ & $45 \mathrm{nmol} / \mathrm{L}$ & $60-150 \mathrm{nmol} / \mathrm{L}$ \\
$\mathrm{TSH}$ & 4,12 & $0,5-4 \mathrm{mlU} / \mathrm{L}$ \\
\hline
\end{tabular}

Tras haberse constatado el diagnóstico mediante los datos clínicos y los exámenes de laboratorio, se colocó una sonda nasogástrica (levine) y una sonda uretral para cuantificar la orina y realizar el balance hidromineral de la paciente; se le administraron 2 bulbos de sulfato de $\mathrm{Mg}$ (10\%) diluidos en $500 \mathrm{ml}$ de solución salina 0,9\% durante 12 horas; se requirió la administración de cefotaxima (bulbo 1g), 1 bulbo endovenoso cada 8 horas como profilaxis, y el tratamiento sustitutivo hormonal con levotiroxina (0,1 mg), 1 tableta cada 12 horas. Tras una semana de tratamiento, se fueron observando progresivamente mejorías en cuanto al estado físico y de conciencia de la paciente, con reversión del cuadro clínico. Afortunadamente, fue egresada a los 24 días de su ingreso, con una valoración pendiente del endocrinólogo para la terapia sustitutiva ambulatoria.

\section{Discusión}

El diagnóstico de esta complicación es intensamente complejo, tratándose de una entidad de extraña aparición, registrándose hasta 2006 solamente 300 casos. $^{10}$ Daña diversos sistemas, siendo el cardiovascular el más afectado. Produce menor gasto cardíaco, mediado por disminución de la frecuencia y contractibilidad cardíaca. Además, existe un desbalance en la actividad adrenérgica, con predominancia de la función alfa, por menor actividad de los receptores beta, lo que genera un aumento de la resistencia vascular periférica. La enfermedad se asocia con menor respuesta ventilatoria, hipercapnia e hipoxemia, anemia normocítica y aumento de la creatinina plasmática, todos los cuales generan una mayor morbimortalidad.

El diagnóstico de esta entidad nosológica se realizó teniendo en cuenta la epidemiología de la enfermedad, signos y síntomas y exámenes complementarios, en los cuales se evidenciaron alteraciones frecuentes de dicha complicación. Es fundamental aclarar que, en nuestro centro, los exámenes complementarios correspondientes al perfil tiroideo no son realizados por el Servicio de Urgencias, lo que provocó que se hicieran cuando se había emprendido la terapia hormonal empírica con levotiroxina, y esto pudo haber alterado los valores reales.

En una investigación realizada por el doctor Ray Ticse, de la Universidad Peruana "Cayetano Heredia", de Lima, se confirmó que el coma mixedematoso está asociado a trastornos en la reabsorción de electrolitos, disminución del filtrado glomerular y del flujo plasmático, ${ }^{4}$ lo que concuerda totalmente con nuestro estudio.

Andrés Domínguez Borgúa, en un estudio realizado en el Hospital Regional de Tlalnepantla, en México, confirmó la existencia del cuadro clínico típico del coma mixedematoso presentado en este caso, ${ }^{12}$ aunque se notaron algunas diferencias con respecto a la investigación realizada en dicho centro, como la existencia de rectorragia y cianosis central y periférica.

Un artículo perteneciente a la Revista de la Asociación Mexicana de Medicina Crítica y Terapia Intensiva, refleja al coma mixedematoso como una causa de extubación fallida, 


\section{Coma mixedematoso sin diagnóstico de hipotiroidismo / Albín-Cano et al}

conducente a una insuficiencia respiratoria, en una paciente con clínica de dicha patología y que obligó a su reintubación. ${ }^{13}$ Este caso clínico corrobora las manifestaciones respiratorias de nuestra paciente, por coincidencia con la acidosis respiratoria, lo que demuestra la repercusión marcada que puede tener dicha afección en los diferentes sistemas.

Es importante tener en cuenta la asociación efectuada por los investigadores Carlos A. Pedroza y Verónica SánchezRíos, acerca de la interacción entre la insuficiencia cardíaca y el coma mixedematoso, al ser la primera una de las principales enfermedades crónicas que afectan a la población, comprobándose, además, que puede comportarse como un factor desencadenante. ${ }^{14}$

La inducción del coma mixedematoso por la amiodarona se observa con cierta periodicidad. Dos estudios reflejan la repercusión de este fármaco antiarrítmico en el desencadenamiento de dicha patología, proponiendo a este medicamento como uno de los factores desencadenantes más influyentes en cuanto al agravamiento del hipotiroidismo, sobre todo por la gran cantidad de yodo que contiene, lo que altera la función tiroidea. ${ }^{15,16}$

El descubrimiento más relevante en este caso es su diagnóstico sin previo conocimiento del antecedente personal de la enfermedad de base. La aparición de trastornos electrolíticos, disminución del filtrado glomerular y del flujo plasmático, comprueban que esta entidad puede concomitar con una insuficiencia renal crónica. Es significativa la evolución favorable del caso, a pesar de haber presentado un cuadro bien establecido, alteraciones humorales importantes y una elevada mortalidad de la enfermedad.

\section{Referencias}

1. Ángeles M. Estructura y función de la glándula tiroides. Rev. ORL, 2016, 7, Supl. 2, pp. 7-16.
2. López ITM, Samón MC, Martínez MM, Anderson JR. Coma mixedematoso en hombre de 74 años: reporte de caso con revisión narrativa. Medwave 2013;13(4)

3. Palacios Moguel Paul, Cepeda Jiménez Miguel, Arellano Estrada Juan Manuel. Coma mixedematoso y síndrome de Ogilvie asociado a aripiprazol, memantina y venlafaxina. Reporte de caso. Med. crít. (Col. Mex. Med. Crít.)2016; 30:3426.

4. Ticse R, Valenzuela C, Villena J, Huachin M, Pinto M, Guillen M, et al. Coma mixedematoso e insuficiencia renal: Reporte de casos y revisión de la literatura. Rev Med Hered. 2011; 22:82-5.

5. Meléndez GAG, Betanzos RR, Pedraza VS, Palomo AS, Hernández CFM, Montaño SA. Hypothyroidism. Med Int Méx 2010;26:462-71.

6. Sasazawa DT, Tsukumo DM, Lalli CA. Myxedema coma in a patient with type 1 neurofibromatosis: rare association. Arq Bras Endocrinol Metab. 2013;57:743-7.

7. Leal Curí L. Coma mixedematoso. Rev Cub End. 2012; 23:273-80.

8. Rizzo LF, Mana DL, Bruno OD, Wartofsky L. Coma mixedematoso. Medicina (Buenos Aires). 2017;77.

9. Mathew V, Misgar RA, Ghosh S, Mukhopadhyay P, Roychowdhury P, Pandit $\mathrm{K}$, et al. Myxedema coma: a new look into an old crisis. Journal of thyroid research. 2011;2011.

10. Rebollo-Gómez H. Coma mixedematoso. Informe de un caso. Rev Med Inst Mex Seguro Soc 2010; 48 : 215-217.

11. Nazar C, Bastidas J, Zamora M, Coloma R, Fuentes R. Manejo perioperatorio de pacientes con patología tiroidea y tratamiento crónico con corticoides. Rev Chil Cir. 2016;68:87-93.

12. Domínguez-Borgúa Andrés, Fonseca-Entzana Marcos T, Trejo-Martínez Miguel A. Coma Mixedematoso. Med Int Méx 2015; 31: 1.

13. Romero IIT, Robledo GC, Maldonado PÁ, Núñez Pérez-Redondo C, Vargas CW, Cicero Sabido R. Hipotiroidismo y extubación fallida. Comentario breve a propósito de un caso. Rev Asoc Mex Med Crit y Ter Int 2013;27:115-118.

14. Pedroza-Mosquera CA, Sánchez-Ríos V, Solarte-Bastidas YA, Vallejo S. Coma mixedematoso e insuficiencia cardíaca. Reporte de un caso clínico. Rev Mex Endocrinol Metab Nutr. 2017;4:188-91.

15. Salinas Arce JL, Pinto Valdivia M, Solórzano Altamirano P. Hipotiroidismo inducido por Amiodarona: Reporte de caso. Rev Med Hered. 2012;23:45-7.

16. Casuso E, Nan D, Hernández JL, Regata OG, Alonso J, González-Macías J. Disfunción tiroidea inducida por amiodarona. Análisis en condiciones de práctica clínica habitual. Med Int Méx. 2011;27:231-7. 